Пронин А. И., Щелкунов Е. Б., Султангареева А. С., Латышев К. А., Иваненко А. А. A. I. Pronin, Ye. B. Shchelkunov, A. Ye. Sultangareeva, K. A. Latyshev, A. A. Ivanenko ИССЛЕДОВАНИЕ И РАЗРАБОТКА КОНСТРУКЦИИ ДЕТАЛИ КРОНШТЕЙН С ИСПОЛЬЗОВАНИЕМ САD/САЕ СИСТЕМ

\title{
RESEARCH AND WORKING OUT OF THE BRACKET PART WITH CAD/CAE SYSTEMS
}

Пронин Александр Иннокентьевич - кандидат технических наук, доцент кафедры «Технология машиностроения» ФГБОУ ВО «Комсомольский-на-Амуре государственный технический университет» (Россия, г. Комсомольск-на-Амуре). E-mail: mdsov@knastu.ru.

Mr. Alexandre I. Pronin - PhD in Engineering, Associate Professor, Mechanical Engineering Department, Komsomolsk-on-Amur State Technical University (Russia, Komsomolsk-on-Amur). E-mail: mdsov@knastu.ru.

Щелкунов Евгений Борисович - кандидат технических наук, доцент кафедры «Технология машиностроения» ФГБОУ ВО «Комсомольский-на-Амуре государственный технический университет» (Россия, г. Комсомольск-на-Амуре). E-mail: ktm@knastu.ru.

Mr. Yevgeny B. Shchelkunov - PhD in Engineering, Associate Professor, Mechanical Engineering Department, Komsomolsk-on-Amur State Technical University (Russia, Komsomolsk-on-Amur). E-mail: ktm@knastu.ru.

Султангареева Алеся Евгеньевна - студентка кафедры «Технология машиностроения» ФГБОУ ВО «Комсомольский-на-Амуре государственный технический университет» (Россия, г. Комсомольск-наАмуре). E-mail: kmopevm@knastu.ru.

Ms. Alesya Ye. Sultangareeva - a student, Mechanical Engineering Department, Komsomolsk-on-Amur State Technical University (Russia, Komsomolsk-on-Amur). E-mail: kmopevm@knastu.ru.

Латышев Кирилл Андреевич - студент кафедры «Технология металлов» ФГБОУ ВО «Комсомольскийна-Амуре государственный технический университет» (Россия, г. Комсомольск-на-Амуре).

Mr. Kirill A. Latyshev - a student, Metal Technology Department, Komsomolsk-on-Amur State Technical University (Russia, Komsomolsk-on-Amur).

Иваненко Ангелина Александровна - студентка кафедры «Технология машиностроения» ФГБОУ ВО «Комсомольский-на-Амуре государственный технический университет» (Россия, г. Комсомольск-наАмуре).

Ms. Angelina A. Ivanenko - a student, Mechanical Engineering Department, Komsomolsk-on-Amur State Technical University (Russia, Komsomolsk-on-Amur).

Аннотация. В современных условиях жесткой конкуренции машиностроительные предприятия стремятся максимально использовать широкие возможности компьютеров в решении задач проектирования и производства изделий. Для автоматизации этих задач используются СAD/CAM/CAE системы. Цель работы произвести экспресс-анализ детали кронштейн, заключающийся в оценке прочности разработанной конструкции по допускаемым напряжениям, определении наиболее слабых мест конструкции, возможности внесения необходимых изменений (оптимизации) в изделия. В качестве объекта исследования использовалась 3D модель детали кронштейн. Для проведения экспресс-анализа детали кронштейн использовался T-FLEX Анализ - это интегрированная с T-FLEX CAD среда конечно-элементных расчётов. Модуль выполняет расчет на прочность конструкции под действием приложенных к системе сил. Результатом работы разработчика в модуле является получение оценки прочности спроектированной им конструкции по допускаемым напряжениям, определение слабых мест конструкции и изменение конструкции изделия, т.е. его оптимизация. Анализ результатов показал, что выполненные в результате изменения в конструкции детали привели к уменьшению модуля перемещений, эквивалентных напряжений, возникающих в детали, эквивалентных деформаций и привели к существенному увеличению коэффициента запаса по эквивалентным напряжениям.

Summary. In modern severe competition, engineering enterprises seek to maximize opportunities to use computers in designing and manufacturing products. To automatize these tasks one can use CAD/CAM/SAE systems. The 
purpose of the work is to make rapid analysis of the bracket parts, which supposes assessing the durability of the developed design for allowable stresses, the determination of the weakest spots in the structure, the possibility of making the necessary changes in the product. A 3D model bracket parts present the object of investigation. For the rapid analysis of the bracket parts, the authors used T-FLEX Analysis that is an environment of finite-element calculations integrated with T-FLEX CAD. The module performs the calculation of the structure durability under the influence of forces applied to the system. The aim of the researcher in the module is to provide an estimation of durability of the developed design for the allowable stresses, the determination of the weakest spots in the structure and changes in the product, i.e. optimization. The analysis of the results showed that performed changes in the construction of parts have led to a decrease in the displacement module that is equivalent to stresses arising in the part, the equivalent strain and a significant increase of the equivalent stress.

Ключевые слова: метод конечных элементов, твердотельная модель, деталь, прочность, напряжение, статический анализ, конструкция, оптимизация, жесткость.

Key words: finite-element method, solid-state model, part, durability, voltage, static assaying, cconstruction, optimization, hardness.

УДК 621.9

\section{Введение}

В современных условиях жесткой конкуренции машиностроительные предприятия стремятся максимально использовать широкие возможности компьютеров в решении задач проектирования и производства изделий. Для автоматизации этих задач используются CAD/CAM/CAE системы. Достижению высоких показателей качества изделий способствует широкое использование САЕ-систем, которые ещё на стадии возникновения идеи о создании объекта производства и принятия предпроектных решений позволяют разработчику произвести целый ряд исследований. Можно моделировать условия работы создаваемого изделия и оценивать показатели, характеризующие технические возможности будущего изделия от воздействия различного рода внешних и внутренних факторов. Это особенно важно при создании сложных устройств, натурные испытания которых требуют огромных затрат и могут быть осуществлены только после завершения всех стадий проектирования и производства. Допущенные ошибки при создании таких объектов производства могут обернуться не только коммерческой неудачей, но и трагическими последствиями.

Для решения различных задач инженерных расчетов «Топ системы» предлагает специализированную среду конечно-элементных расчётов T-FLEX Анализ. Она характеризуется глубокой интеграцией с комплексом геометрических редакторов.

Цель работы - произвести экспресс-анализ детали кронштейн, заключающийся в оценке прочности разработанной конструкции по допускаемым напряжениям, определении наиболее слабых мест конструкции, возможности внесения необходимых изменений (оптимизации) в изделия.

\section{Методика проведения исследования}

В качестве объекта исследования использовалась 3D модель детали кронштейн. Деталь кронштейн является базовой деталью сборочного узла (см. рис. 1) и служит шаровой опорой ствола орудия. Она позволяет регулировать положение ствола в горизонтальной и вертикальной плоскостях. Сборочный узел является основным несущим узлом и воспринимает силу отдачи при стрельбе. Разработка 3D модели выполнялась в CAD системе T-FLEX CAD.

Для проведения экспресс-анализа детали кронштейн использовался модуль системы TFLEX Анализ. В модуле T-FLEX Анализ выполнялось математическое моделирование возникающих в процессе эксплуатации физических явлений, решались значимые задачи. В основе расчетов лежал метод конечных элементов. Вносимые изменения в геометрическую модель (параметрическую) автоматически переносились на сеточную конечно-элементную модель. 


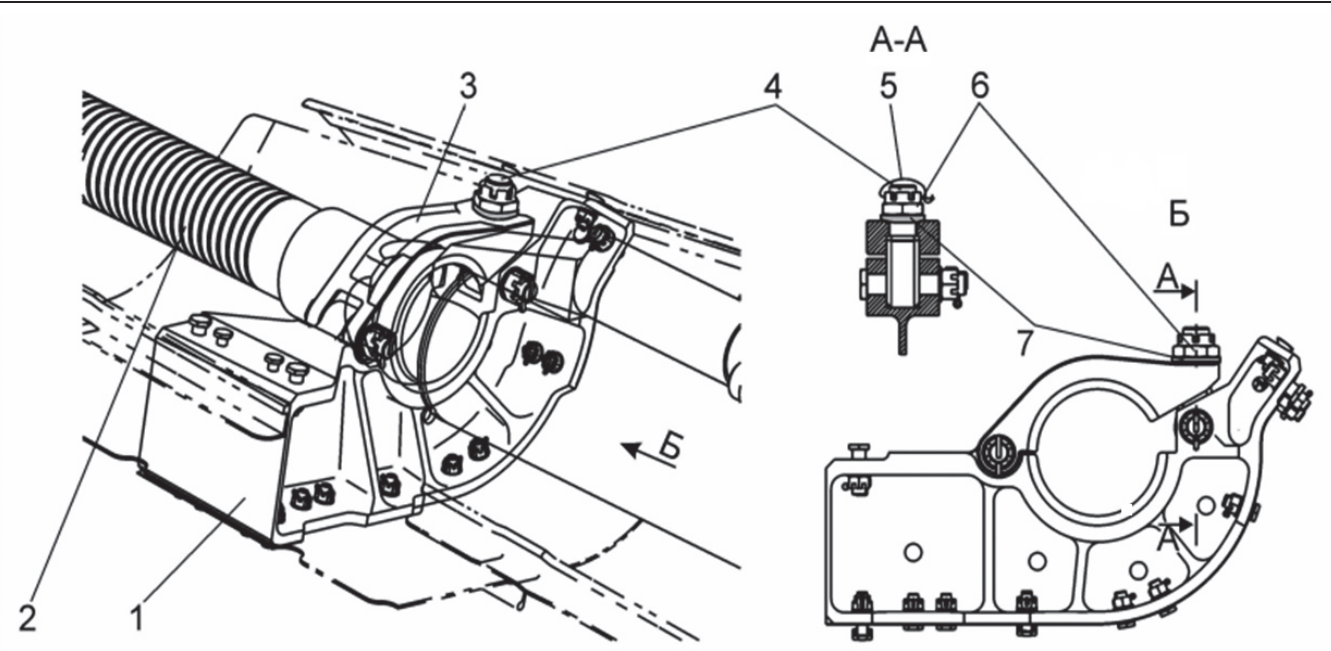

Рис. 1. Сборочный узел: 1 - кронштейн; 2 - ствол орудия; 3 - верхний хомут; 4 - серьга; 5 - контровочная булавка; 6 - прижимная гайка; 7 - сферическая гайка

В модуле выполнялся расчёт напряжённого состояния конструкции детали кронштейн под действием постоянных во времени сил, приложенных к системе. Расчет слабых мест детали и последующая оптимизация конструкции детали выполнялись по следующей методике $[1 ; 2 ; 3]$. Создавалась тетраэдальная конечно-элементная модель детали. Задавались граничные условия для расчета на прочность. Функцию граничных условий в статике выполняют закрепления и приложенные к системе внешние нагрузки. Формировалась система линейных алгебраических уравнений, выполнялось их решение. Опции расчета в диалоге свойства - задачи изменялись разработчиком самостоятельно. Результаты расчета отображались в дереве задач. Распределение сил воздействия оценивалось по цвету: красный показывает наибольшее воздействие, синий - наименьшее. Визуализация результатов осуществлялась в отдельном 3D окне T-FLEX CAD. Одновременно открывались несколько окон с результатами одной или разных задач. Разработчиком создавались независимые от T-FLEX Анализа электронные документы, содержащие основные сведения о рассчитанной задаче. Отчёт формировался в html-формате. Отчёт содержал основные сведения о модели, материалах, расчётной конечно-элементной сетке, а также цветные эпюры результатов, которые отображались в дереве задач или открывались в окнах визуализатора.

\section{Результаты исследования и их обсуждение}

На рис. 2 представлена тетраэдальная конечно-элементная модель изделия. Выполнялась генерация сетки 3D модели. Относительный размер сетки менялся с целью добиться однородности распределения формы образующих сетку элементов. Относительный размер сетки равен 0,04 . Стрелками показаны сгущения конечных элементов в модели, в которых можно ожидать концентрации напряжений.

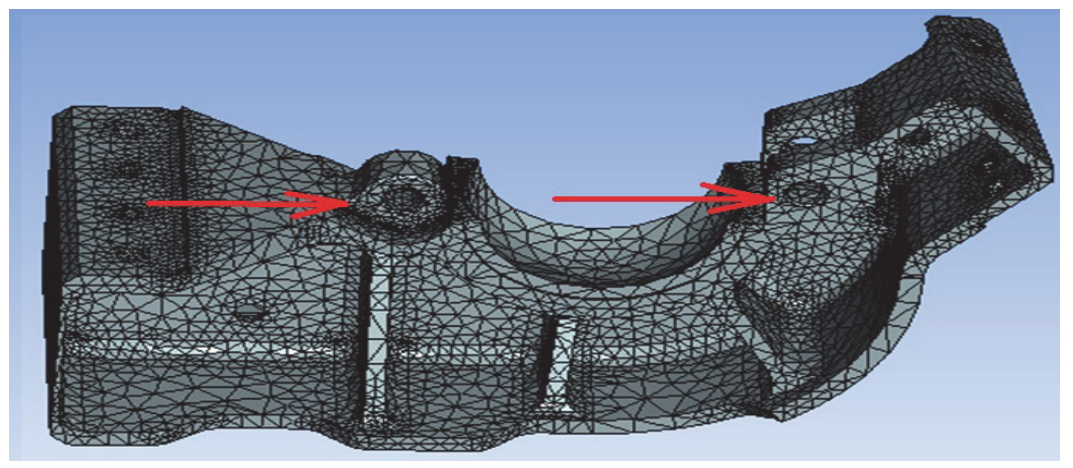

Рис. 2. Тетраэдальная конечно-элементная модель изделия 


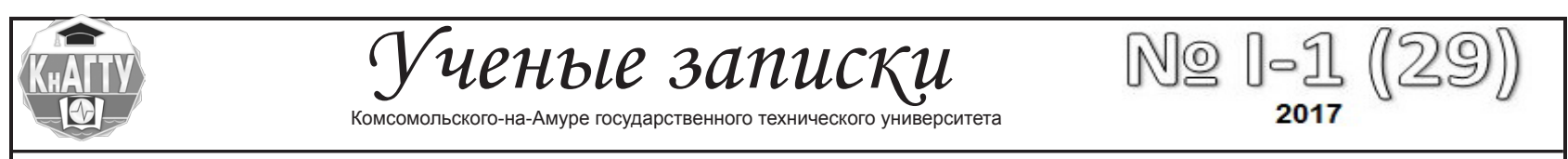

Для расчета на прочность были заданы граничные условия. Функцию граничных условий в статике выполняют закрепления и приложенные к системе силы воздействия (см. рис. 3).

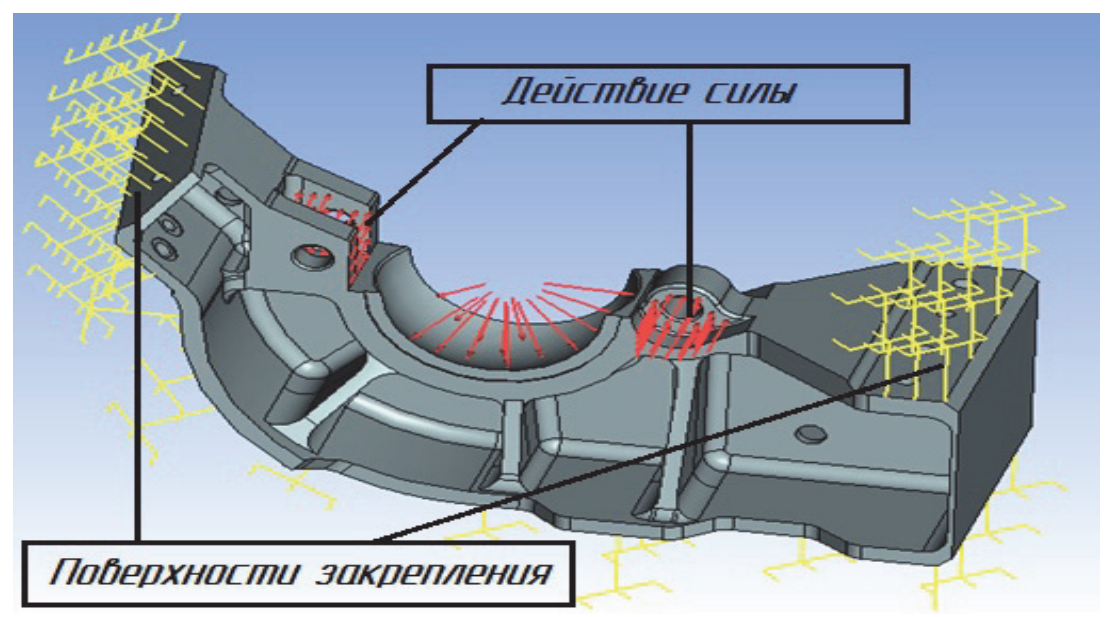

Рис. 3. Назначение условий закрепления и нагружения

Созданные силы равномерно распределялись по указанным граням детали. Силы направлены перпендикулярно к нагружаемым граням детали.

Из рис. 4 - 5 видно, что наибольшие по модулю перемещения будут иметь грани проушин кронштейна, а максимальные эквивалентные напряжения возникнут в месте крепления готового изделия. В полнофункциональной версии «Экспресс-анализ» были получены численные значения перемещений и напряжений, позволяющие изменить размеры кронштейна, добившись требуемой прочности и жесткости детали.

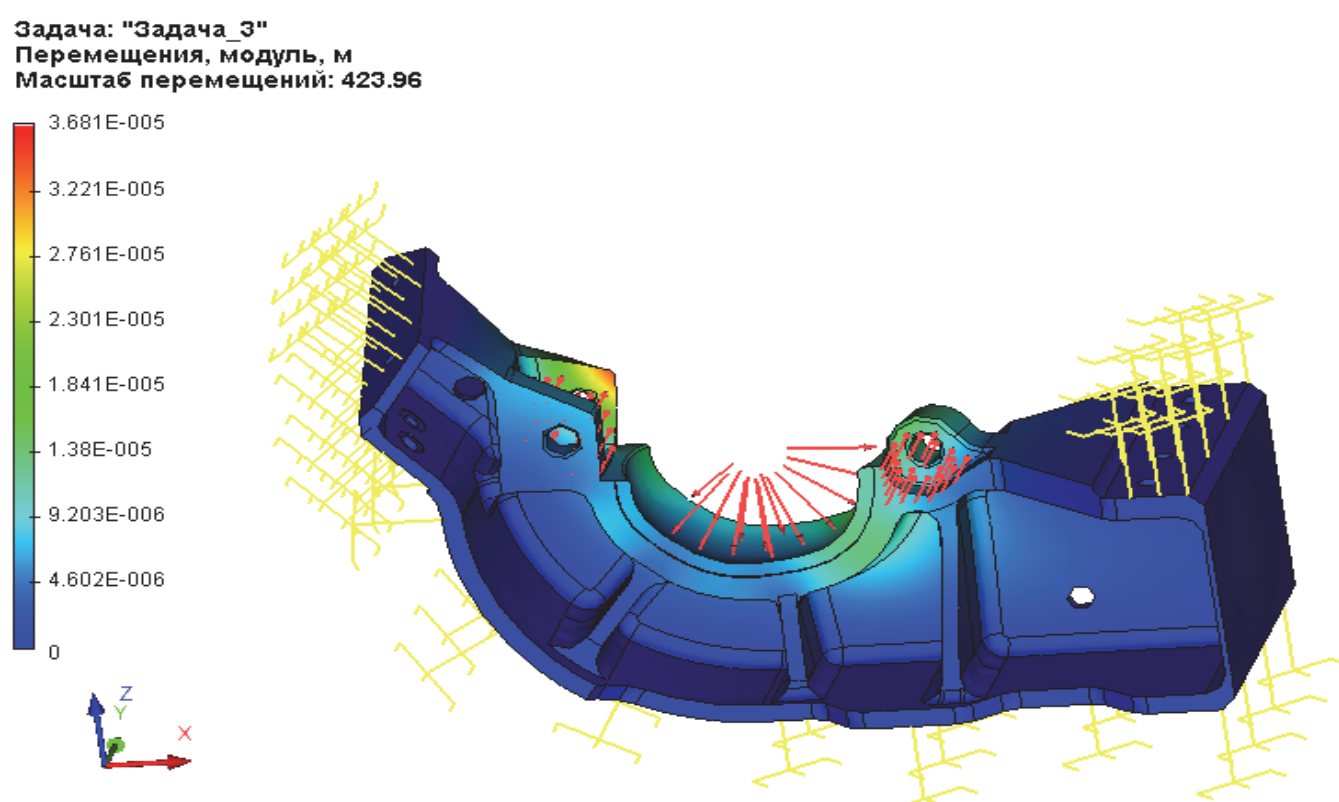

Рис. 4. Эпюра модуля перемещения 
ПрОНИН А. И., ЩЕЛКУНОв Е. Б., СУЛТАНГАРЕЕВА А. С., ЛАТЫШЕВ К. А., ИвАНЕНКо А. А.

ИССЛЕДОВАНИЕ И РАЗРАБОТКА КОНСТРУКЦИИ ДЕТАЛИ КРОНШТЕЙН С ИСПОЛЬЗОВАНИЕМ САD/САЕ СИСТЕМ

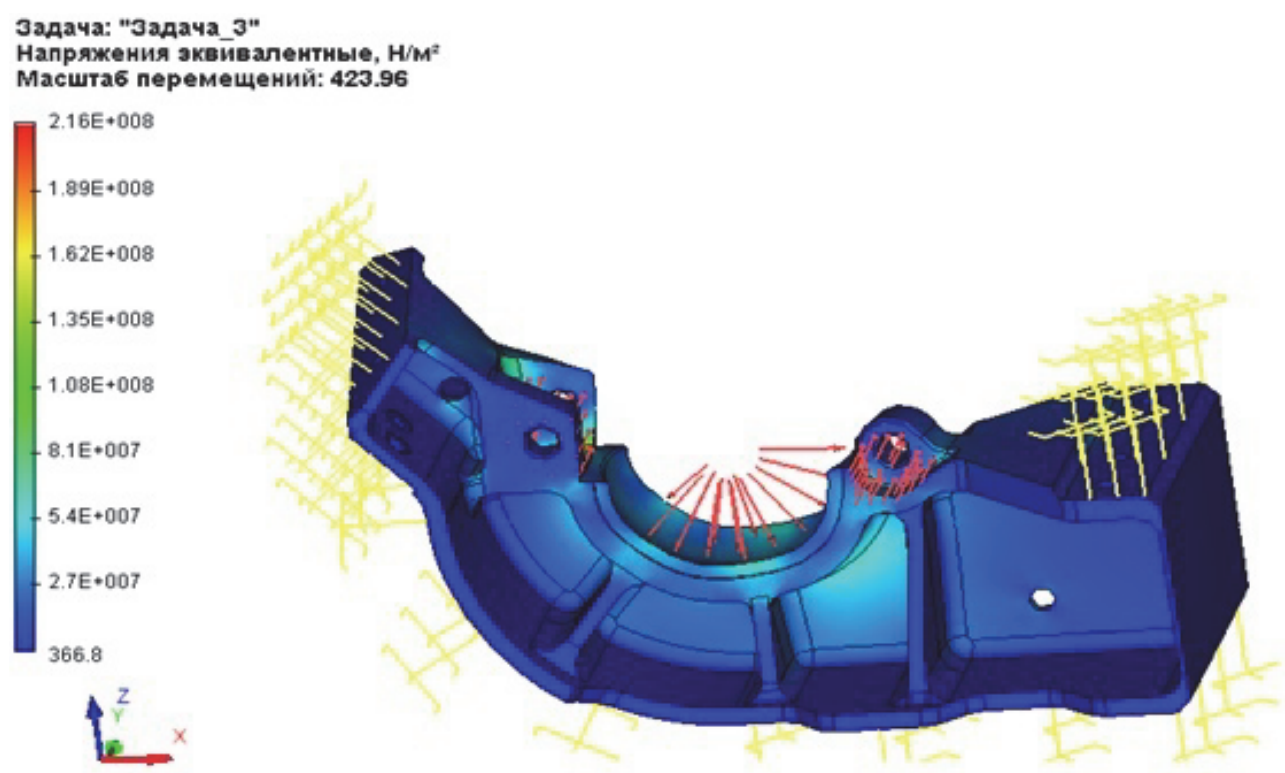

Рис. 5. Эпюра эквивалентных напряжений

На рис. 6 показана эпюра коэффициента запаса прочности по эквивалентным напряжениям. При этом после выбора коэффициента запаса по эквивалентным напряжениям в служебном окне «Задачи» укажем в окне «Параметры эпюры - коэффициент запаса», что запас прочности должен определяться по пределу текучести материала детали. Из результатов анализа можно сделать вывод о том, что кронштейн нагружен. Такие элементы детали, как «проушины», сильно нагружены. Если коэффициент запаса меньше 1 , то произойдет разрушение.

Из рис. 4 - 6 видно, что наименее нагруженными являются ребра и основания кронштейна.

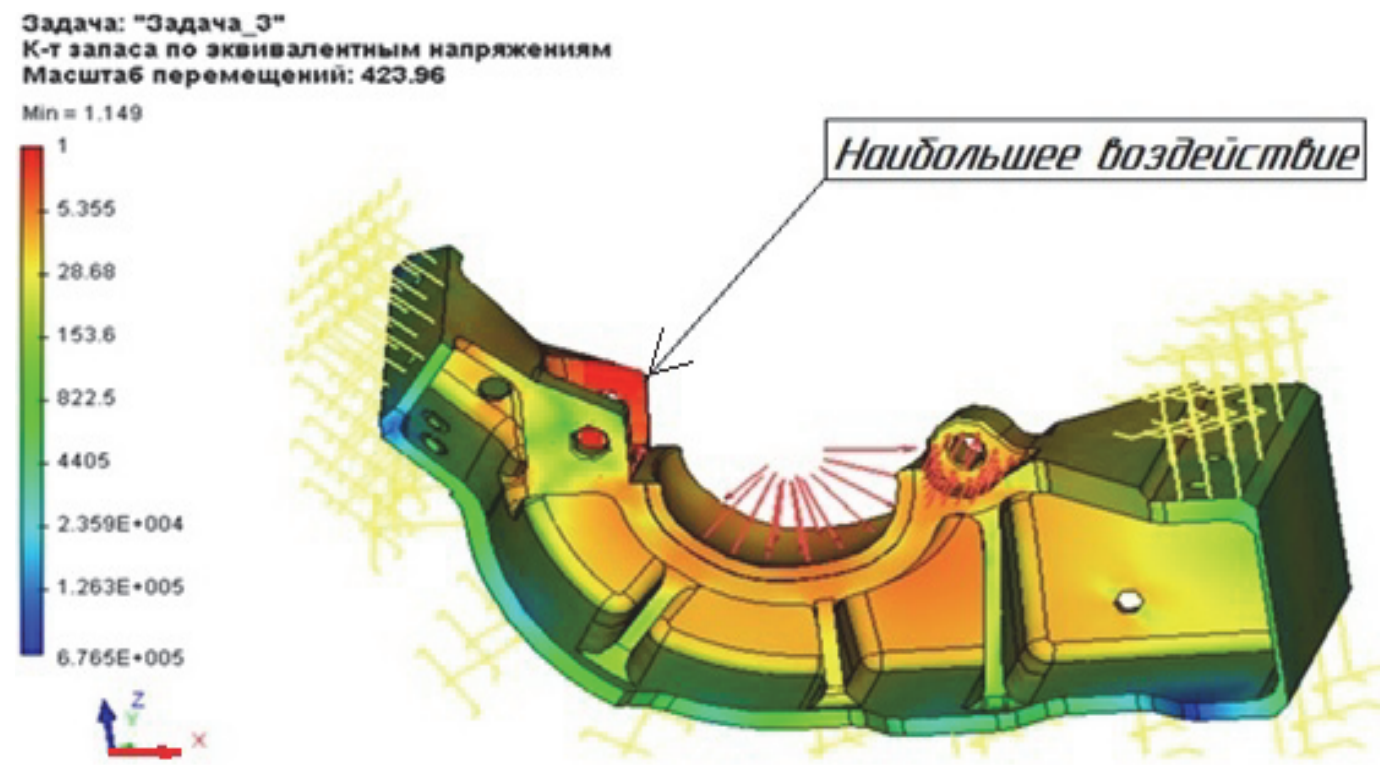

Рис. 6. Эпюра коэффициента запаса прочности по эквивалентным напряжениям

Оценив полученные результаты, делаем вывод, что необходимо оптимизировать конструкцию детали «Кронштейн». Было предложено увеличить на 4 мм толщину стенки «проушин». Конструкция детали после доработки была подвергнута статическому анализу. 


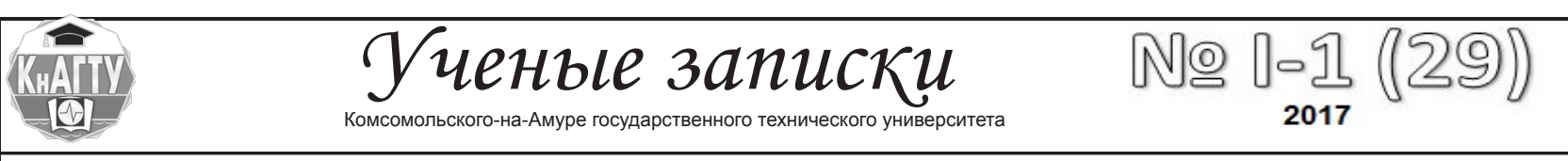

После переразбиения модели вновь запустим расчет. Результаты анализа для модифицированной модели кронштейна приведены на рис. 7.

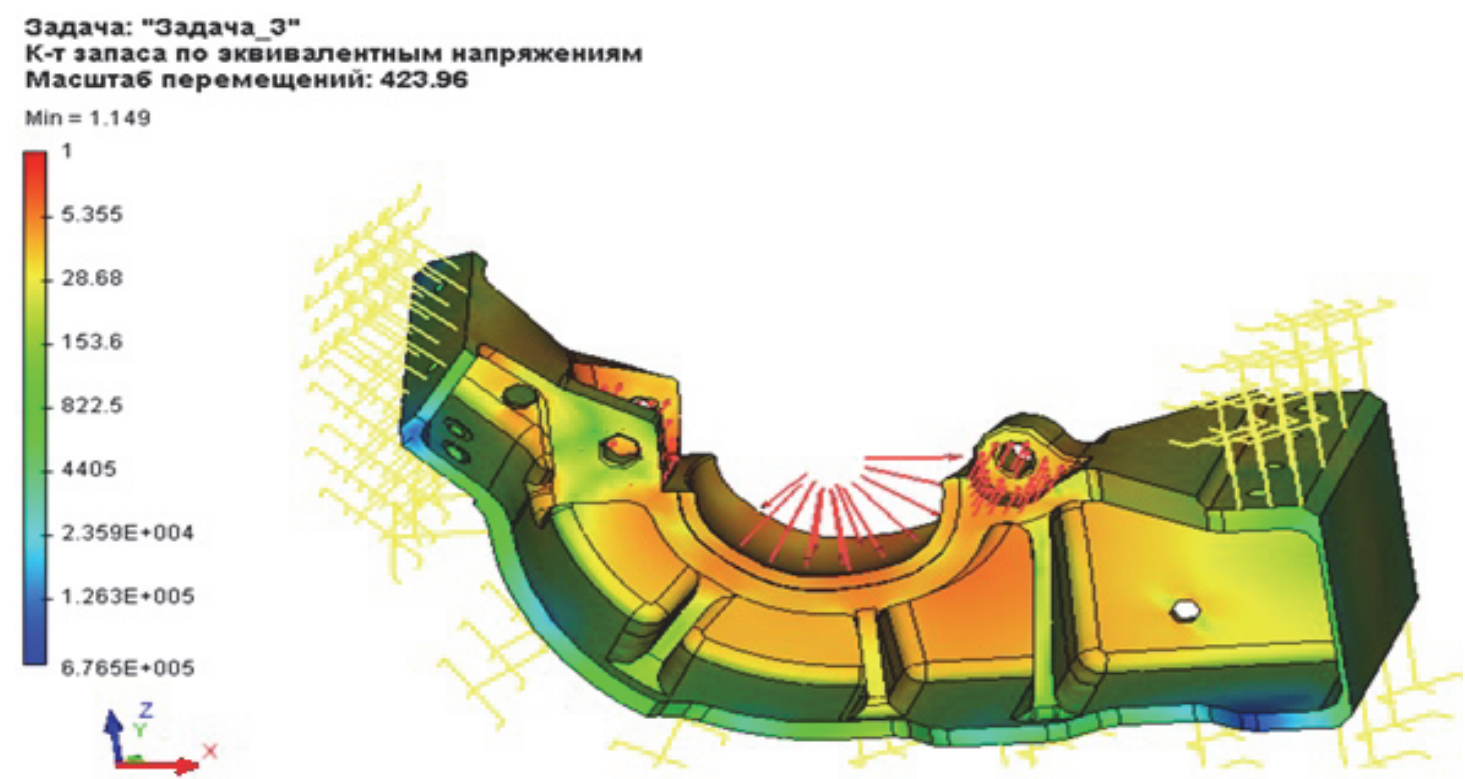

Рис. 7. Эпюра коэффициента запаса прочности по эквивалентным напряжениям

Анализ результатов отчета (см. рис. 7), показывает, что существенно улучшились характеристики коэффициента запаса по эквивалентным напряжениям. Таким образом, задачу оптимизации конструкции можно считать выполненной.

\section{Заключение}

Выполненный анализ позволил осуществить расчет напряженного состояния конструкции детали «кронштейн» под действием приложенных к ней сил с помощью модуля «Статический анализ». Была произведена оценка прочности разработанной конструкции по допускаемым напряжениям, определены наиболее слабые места конструкции, изменены размеры исследуемого изделия, обновлена конечно-элементная модель и сразу же получены результаты расчёта измененной модели. Данный анализ позволил в короткие сроки просчитать несколько вариантов конструкции и выбрать из них оптимальный.

\section{ЛИТЕРАТУРА}

1. Киричек, А. В. Проектирование металлорежущих инструментов и технологической оснастки в T-FLEX CAD: учеб. пособие / А. В. Киричек, А. Н. Афонин. - М.: Машиностроение-1, 2007. - 158 с.

2. Устройство для ориентации заготовок / Е. Б. Щелкунов, С. В. Виноградов, М. Е. Щелкунова, А. И. Пронин // Ученые записки Комсомольского-на-Амуре государственного технического университета. Науки о природе и технике. - Комсомольск-на-Амуре: ФГБОУ ВО «КнАГТУ». - 2016. - № II-1(24). - С. 29-33.

3. Рулева, Н. Е. Современное направление в машиностроении - высокоскоростная обработка / Н. Е. Рулева // Международный журнал «Научный взгляд в будущее». - 2016. - Выпуск 2(2). - С. 133а. 